\title{
$W^{a}$ Ve phenomena
}

\section{Biharmonic wave maps into spheres}

Sebastian Herr, Tobias Lamm, Roland Schnaubelt

CRC Preprint 2018/45, December 2018

\section{KARLSRUHE INSTITUTE OF TECHNOLOGY}

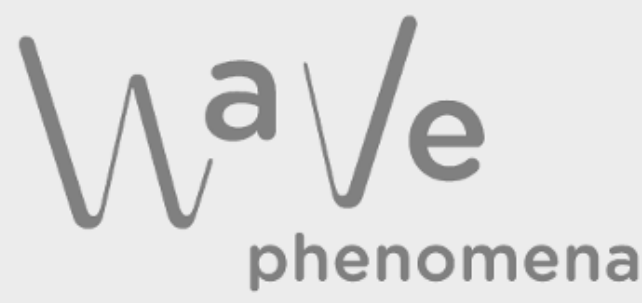

CRC 1173 


\section{Participating universities}

EBERHARD KARLS

UNIVERSITATT TUBINGEN

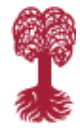

Funded by

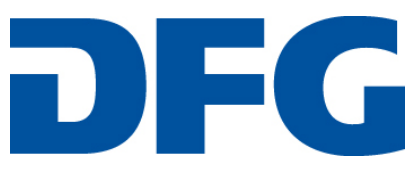

ISSN 2365-662X 


\title{
BIHARMONIC WAVE MAPS INTO SPHERES
}

\author{
SEBASTIAN HERR, TOBIAS LAMM, AND ROLAND SCHNAUBELT
}

\begin{abstract}
A global weak solution of the biharmonic wave map equation in the energy space for spherical targets is constructed. The equation is reformulated as a conservation law and solved by a suitable Ginzburg-Landau type approximation.
\end{abstract}

\section{INTRODUCTION}

We study biharmonic wave maps $u: I \times \mathbb{R}^{n} \rightarrow S^{l}$, where $S^{l}$ is the $l$-dimensional unit sphere in $\mathbb{R}^{l+1}$, and $I \subset \mathbb{R}$ is an open interval. These maps are critical points of the action functional

$$
\Phi(u):=\frac{1}{2} \int_{I \times \mathbb{R}^{n}}\left(\left|\partial_{t} u\right|^{2}-|\Delta u|^{2}\right) d(t, x)
$$

acting on functions with values in $S^{l}$. Here $\Delta u=\left(\Delta u^{i}\right)_{i}$ is the extrinsic Laplacian; i.e., the Laplacian w.r.t. $x$ when considering $u$ as a map into $\mathbb{R}^{l+1}$. In our main Theorem 1.1 we construct a global weak solution for all data in the energy space.

We introduce two equivalent versions of the biharmonic wave map system for regular solutions. Sufficiently smooth critical points $u: I \times \mathbb{R}^{n} \rightarrow S^{l}$ of $\Phi$ satisfy

$$
\left(\partial_{t}^{2}+\Delta^{2}\right) u \perp T_{u} S^{l}
$$

which can be viewed as the geometric version of biharmonic wave map equation To show this claim, for $\varphi \in C_{c}^{\infty}\left(I \times \mathbb{R}^{n}, \mathbb{R}^{l+1}\right)$ we consider the variation $u_{\tau}=\pi(u+\tau \varphi)$, where $\pi: \mathbb{R}^{l+1} \backslash\{0\} \rightarrow S^{l}$ denotes the retraction $\pi(y)=\frac{y}{|y|}$, and $\tau>0$ is small enough. We compute

$$
\begin{aligned}
\left.\frac{\mathrm{d}}{\mathrm{d} \tau} \Phi\left(u_{\tau}\right)\right|_{\tau=0} & =\int_{I \times \mathbb{R}^{n}}\left\langle\partial_{t} u, \partial_{t} D \pi(u) \varphi\right\rangle-\langle\Delta u, \Delta D \pi(u) \varphi\rangle d(t, x) \\
& =-\int_{I \times \mathbb{R}^{n}}\left\langle\partial_{t}^{2} u+\Delta^{2} u, D \pi(u) \varphi\right\rangle d(t, x)
\end{aligned}
$$

Choose a smooth orthonormal frame $\left\{v_{1}(t, x), \ldots, v_{l}(t, x)\right\}$ for $T_{u(t, x)} S^{l}$, a scalar function $\eta \in C_{c}^{\infty}\left(I \times \mathbb{R}^{n}, \mathbb{R}\right)$ and define $\varphi_{j}=v_{j} \eta$, for $j=1, \ldots, l$. Since $D \pi(u) \varphi_{j}=$ $\varphi_{j}$, for a critical point $u$ of $\Phi$ we obtain

$$
0=\int_{I \times \mathbb{R}^{n}}\left\langle\partial_{t}^{2} u+\Delta^{2} u, v_{j}\right\rangle \eta d(t, x) .
$$

We conclude that $\left\langle\partial_{t}^{2} u+\Delta^{2} u, v_{j}\right\rangle=0$ for any $j=1, \ldots, l$, which shows (1.1).

Date: December 10, 2018.

2000 Mathematics Subject Classification. 35L75, 58J45.

Key words and phrases. Global weak solutions, geometric plate equation, conservation law.

TL and RS gratefully acknowledge financial support by the Deutsche Forschungsgemeinschaft (DFG) through CRC 1173. 
For smooth $u: I \times \mathbb{R}^{n} \rightarrow S^{l}$, equation (1.1) is equivalent to the PDE-version

$$
\left(\partial_{t}^{2}+\Delta^{2}\right) u=\left[|\Delta u|^{2}-\left|\partial_{t} u\right|^{2}-\Delta|\nabla u|^{2}-2 \operatorname{div}\langle\Delta u, \nabla u\rangle\right] u
$$

of the biharmonic wave map system. Here and below, for any $A \in \mathbb{R}^{(l+1) \times(l+1)}$ the expression $\operatorname{div}\langle\Delta u, A \nabla u\rangle$ is shorthand for $\sum_{i=1}^{n} \partial_{i}\left\langle\Delta u, A \partial_{i} u\right\rangle$, where $\langle\cdot, \cdot\rangle$ is the scalar product in $\mathbb{R}^{l+1}$. We also write $\langle\nabla \Delta u, \nabla u\rangle=\sum_{i=1}^{d}\left\langle\partial_{i} \Delta u, \partial_{i} u\right\rangle$ etc.. Moreover, $|\cdot|$ denotes the Euclidean norm in $\mathbb{R}^{l+1}$ and in $\mathbb{R}^{(l+1)(l+1)}$.

We show the above mentioned equivalence. Equation (1.1) means that there is a function $\lambda_{u}: I \times \mathbb{R}^{n} \rightarrow \mathbb{R}$ such that $\left(\partial_{t}^{2}+\Delta^{2}\right) u=\lambda_{u} u$. A solution to (1.2) of course satisfies this identity with $\lambda_{u}=[\ldots]$. To see the converse, we multiply $\left(\partial_{t}^{2}+\Delta^{2}\right) u=\lambda_{u} u$ by $u$ and use the product rule. It follows that

$$
\lambda_{u}=\left\langle\partial_{t}^{2} u, u\right\rangle+\left\langle\Delta^{2} u, u\right\rangle=\partial_{t}\left\langle\partial_{t} u, u\right\rangle-\left\langle\partial_{t} u, \partial_{t} u\right\rangle+\operatorname{div}\langle\nabla \Delta u, u\rangle-\langle\nabla \Delta u, \nabla u\rangle .
$$

By $|u|^{2}=1$, we have $2\left\langle\partial_{k} u, u\right\rangle=\partial_{k}|u|^{2}=0$ for $k \in\{t, 1, \ldots, n\}$. We then compute

$$
\begin{aligned}
\lambda_{u} & =-\left|\partial_{t} u\right|^{2}+\Delta\langle\Delta u, u\rangle-2 \operatorname{div}\langle\Delta u, \nabla u\rangle+\langle\Delta u, \Delta u\rangle \\
& =-\left|\partial_{t} u\right|^{2}+\Delta \operatorname{div}\langle\nabla u, u\rangle-\Delta\langle\nabla u, \nabla u\rangle-2 \operatorname{div}\langle\Delta u, \nabla u\rangle+|\Delta u|^{2} \\
& =|\Delta u|^{2}-\left|\partial_{t} u\right|^{2}-\Delta|\nabla u|^{2}-2 \operatorname{div}\langle\Delta u, \nabla u\rangle,
\end{aligned}
$$

as asserted.

The energy corresponding to $\Phi$ is given by

$$
E[u](t)=\frac{1}{2} \int_{\{t\} \times \mathbb{R}^{n}}\left(\left|\partial_{t} u\right|^{2}+|\Delta u|^{2}\right) d x .
$$

We thus introduce the space for (global) weak solutions of our problems as

$$
Z=\left\{u \in L^{\infty}\left(\mathbb{R} \times \mathbb{R}^{n}, \mathbb{R}^{l+1}\right) \mid u(t, x) \in S^{l} \text { a.e., } \partial_{t} u, \Delta u \in L_{\mathrm{loc}}^{2}\left(\mathbb{R} ; L^{2}\left(\mathbb{R}^{n}, \mathbb{R}^{l+1}\right)\right)\right\} .
$$

As above, one observes that

$$
\langle\Delta u, u\rangle=\operatorname{div}\langle\nabla u, u\rangle-\langle\nabla u, \nabla u\rangle=-|\nabla u|^{2},
$$

so that each $u \in Z$ satisfies

$$
|\nabla u|^{2} \leq|\Delta u| \quad \text { and } \quad|\nabla u| \in L_{\mathrm{loc}}^{4}\left(\mathbb{R} ; L^{4}\left(\mathbb{R}^{n}\right)\right) .
$$

A weak solution of (1.1) is defined as a map $u \in Z$ fulfilling

$$
0=\int_{\mathbb{R} \times \mathbb{R}^{n}}\left(-\left\langle\partial_{t} u, \partial_{t} v\right\rangle+\langle\Delta u, \Delta v\rangle\right) d t d x
$$

for all functions $v$ belonging to

$V:=\left\{\left.v \in L^{\infty}\left(\mathbb{R} \times \mathbb{R}^{n}, \mathbb{R}^{l+1}\right)|| \partial_{t} v|,| \nabla v\right|^{2},|\Delta v| \in L^{2}\left(\mathbb{R} \times \mathbb{R}^{n}\right) ; \operatorname{supp}_{t} v\right.$ is compact, and $v$ satisfies $v(t, x) \in T_{u(t, x)} S^{l}$ for a.e. $\left.(t, x) \in \mathbb{R} \times \mathbb{R}^{n}\right\}$,

where $\operatorname{supp}_{t}$ denotes the support of $v: \mathbb{R} \rightarrow L^{\infty}\left(\mathbb{R}^{n}, \mathbb{R}^{l+1}\right), t \mapsto v(t, \cdot)$. Moreover, $u \in Z$ is a weak solution of (1.2) if

$$
\begin{aligned}
& \int_{\mathbb{R} \times \mathbb{R}^{n}}\left(-\left\langle\partial_{t} u, \partial_{t} \phi\right\rangle+\langle\Delta u, \Delta \phi\rangle\right) d t d x \\
& =\int_{\mathbb{R} \times \mathbb{R}^{n}}\left(\left(|\Delta u|^{2}-\left|\partial_{t} u\right|^{2}\right)\langle u, \phi\rangle-|\nabla u|^{2} \Delta\langle u, \phi\rangle+2\langle\Delta u, \nabla u\rangle \nabla\langle u, \phi\rangle\right) d t d x
\end{aligned}
$$

for all $\phi \in W$, where we put

$W:=\left\{\left.v \in L^{\infty}\left(\mathbb{R} \times \mathbb{R}^{n}, \mathbb{R}^{l+1}\right)|| \partial_{t} v|,| \nabla v\right|^{2},|\Delta v| \in L^{2}\left(\mathbb{R} \times \mathbb{R}^{n}\right) ; \operatorname{supp}_{t} v\right.$ is compact $\}$. 
Note that the terms on the right hand side in this definition are integrable by (1.3). In Lemma 2.1 we prove the equivalence of the weak solvability of (1.1) and of (1.2).

The fourth order system (1.2) is analogous to the (second order) wave maps system, see e.g. [7]. In this situation global weak solutions in the energy space have been constructed by Shatah [6] for spherical targets and by Freire [2] for target manifolds being homogeneous spaces. Our main result is a variant of the result of Shatah for biharmonic wave maps.

Theorem 1.1. Let $\left(u_{0}, u_{1}\right) \in L^{\infty}\left(\mathbb{R}^{n}, \mathbb{R}^{l+1}\right) \times L^{2}\left(\mathbb{R}^{n}, \mathbb{R}^{l+1}\right)$ satisfy $\Delta u_{0} \in$ $L^{2}\left(\mathbb{R}^{n}, \mathbb{R}^{l+1}\right)$ as well as $u_{0}(x) \in S^{l}$ and $u_{1}(x) \in T_{u_{0}(x)} S^{l}$ for a.e. $x \in \mathbb{R}^{n}$. Then there is a global weak solution $u \in Z$ of $(1.2)$ with $u(0)=u_{0}$ and $\partial_{t} u(0)=u_{1}$. Moreover, the maps $\Delta u, \partial_{t} u: \mathbb{R} \rightarrow L^{2}\left(\mathbb{R}^{n}, \mathbb{R}^{l+1}\right)$ are continuous and bounded, we have $u(t, \cdot) \in S^{l}$ and $\partial_{t} u(t, \cdot) \in T_{u(t, \cdot)} S^{l}$ a.e. for each $t \in \mathbb{R}$, and the difference $u-u_{0}$ belongs to $C\left(\mathbb{R} ; H^{\theta}\left(\mathbb{R}^{n}, \mathbb{R}^{l+1}\right)\right)$ for all $\theta \in[0,2)$. Finally, for all $t \in \mathbb{R}$ the solution satisfies the energy identity

$$
E[u](t)=\frac{1}{2} \int_{\{t\} \times \mathbb{R}^{n}}\left(\left|\partial_{t} u\right|^{2}+|\Delta u|^{2}\right) d x=E[u](0)=\frac{1}{2} \int_{\mathbb{R}^{n}}\left(\left|u_{1}\right|^{2}+\left|\Delta u_{0}\right|^{2}\right) d x .
$$

As in the case of the above mentioned results for wave maps, our construction uses a suitable Ginzburg-Landau type approximation of (1.2). We refer the reader to [3] for a survey on scalar fourth order wave-type equations.

We note that there is a second functional which also deserves to be called the action functional corresponding to biharmonic wave maps, namely

$\Psi(u):=\frac{1}{2} \int_{\mathbb{R}_{\mathbb{R}^{n}}}\left(\left|\partial_{t} u\right|^{2}-\left|(\Delta u)^{T}\right|^{2}\right) d t d x=\frac{1}{2} \int_{\mathbb{R}_{\mathbb{R}^{n}}}\left(\left|\partial_{t} u\right|^{2}-|\Delta u|^{2}+|\nabla u|^{4}\right) d t d x$,

where $(\Delta u)^{T}=\Delta u+u|\nabla u|^{2}$ is the tangential component of the Laplacian. In this case critical points satisfy the PDE

$$
\partial_{t}^{2} u+\Delta^{2} u+2 \operatorname{div}\left(|\nabla u|^{2} \nabla u\right) \perp T_{u} S^{l}
$$

or equivalently

$$
\begin{aligned}
& \partial_{t}^{2} u+\Delta^{2} u+2 \operatorname{div}\left(|\nabla u|^{2} \nabla u\right)=u\left(|\Delta u|^{2}-\left|\partial_{t} u\right|^{2}-\Delta|\nabla u|^{2}\right. \\
&\left.-2 \operatorname{div}\langle\Delta u, \nabla u\rangle-2|\nabla u|^{4}\right) .
\end{aligned}
$$

Due to the additional nonlinear term, our proof of Theorem 1.1 does not extend to this equation.

\section{The CONSERVATION LAW}

As a first result we show that the systems (1.1) and (1.2) are also equivalent in the weak sense and that they can be can be written in divergence form (2.1). The latter fact will be crucial for our global existence result.

Lemma 2.1. For $u \in Z$ the following assertions are equivalent.

(1) The map $u$ is a weak solution of (1.1).

(2) The map $u$ is a weak solution of (1.2).

(3) For all skew-symmetric matrices $\Lambda \in \mathbb{R}^{(l+1) \times(l+1)}$ the map $u$ is a weak solution of the system

$$
0=\partial_{t}\left\langle\partial_{t} u, \Lambda u\right\rangle+\Delta\langle\Delta u, \Lambda u\rangle-2 \operatorname{div}\langle\Delta u, \Lambda \nabla u\rangle
$$


on $\mathbb{R} \times \mathbb{R}^{n}$ with test functions in $W_{s}$, where

$W_{s}:=\left\{\left.v \in L^{\infty}\left(\mathbb{R} \times \mathbb{R}^{n}\right)|| \partial_{t} v|,| \nabla v\right|^{2},|\Delta v| \in L^{2}\left(\mathbb{R} \times \mathbb{R}^{n}\right) ; \operatorname{supp}_{t} v\right.$ is compact $\}$.

Proof. (1) $\Rightarrow(3)$. Let $u$ be a weak solution of (1.1). Take $\varphi \in V_{s}$ and $\Lambda \in$ $\mathbb{R}^{(l+1) \times(l+1)}$ with $\Lambda^{T}=-\Lambda$. The function $v=\varphi \Lambda u$ belongs to $V$ by (1.3) and takes values in $T_{u} S^{l}$ since $\Lambda$ is skew-symmetric. We thus obtain

$$
\begin{aligned}
0 & =\int_{\mathbb{R} \times \mathbb{R}^{n}}\left(-\left\langle\partial_{t} u, \partial_{t}(\varphi \Lambda u)\right\rangle+\langle\Delta u, \Delta(\varphi(\Lambda u)\rangle) d t d x\right. \\
& =\int_{\mathbb{R} \times \mathbb{R}^{n}}\left(-\partial_{t} \varphi\left\langle\partial_{t} u, \Lambda u\right\rangle+\Delta \varphi\langle\Delta u, \Lambda u\rangle+2 \nabla \varphi\langle\Delta u, \Lambda \nabla u\rangle\right) d t d x
\end{aligned}
$$

using that $\left\langle\partial_{t} u, \Lambda \partial_{t} u\right\rangle=0=\langle\Delta u, \Lambda \Delta u\rangle$. Hence, $u$ is a weak solution of (2.1).

$(3) \Rightarrow(2)$. Let $u$ be a weak solution of (2.1). We employ for $1 \leq i<j \leq l+1$ and $\omega \in S^{l}$ the tangent vectorfields

$$
\Lambda_{i j} \omega=\left(e_{i} \otimes e_{j}-e_{j} \otimes e_{i}\right) \omega=\omega^{i} e_{j}-\omega^{j} e_{i} \in T_{\omega} S^{l}
$$

These vectorfields span $T_{\omega} S^{l}$ since each $\xi \in T_{\omega} S^{l}$ has the representation

$$
\xi=(\omega \otimes \xi-\xi \otimes \omega) \omega=\sum_{1 \leq i<j \leq l+1}\left(\omega^{i} \xi^{j}-\omega^{j} \xi^{i}\right) \Lambda_{i j} \omega .
$$

For a given function $\phi \in W$ we deduce

$$
\begin{gathered}
\phi=\langle\phi, u\rangle u+\sum_{1 \leq i<j \leq l+1} \varphi_{i j} \Lambda_{i j} u, \quad \text { where } \\
\varphi_{i j}:=u^{i}\left(\phi^{j}-\langle\phi, u\rangle u^{j}\right)-u^{j}\left(\phi^{i}-\langle\phi, u\rangle u^{i}\right) .
\end{gathered}
$$

Note that all maps $\varphi_{i j} \Lambda_{i j} u$ and $\langle\phi, u\rangle u$ belong to $W$, and $\varphi_{i j}$ to $W_{s}$. Assertion (3) then yields

$$
\begin{aligned}
& \int_{\mathbb{R} \times \mathbb{R}^{n}}\left(-\left\langle\partial_{t} u, \partial_{t}\left(\varphi_{i j} \Lambda_{i j} u\right)\right\rangle+\left\langle\Delta u, \Delta\left(\varphi_{i j} \Lambda_{i j} u\right\rangle\right) d t d x\right. \\
& =\int_{\mathbb{R} \times \mathbb{R}^{n}}\left(-\partial_{t} \varphi_{i j}\left\langle\partial_{t} u, \Lambda_{i j} u\right\rangle+\Delta \varphi_{i j}\left\langle\Delta u, \Lambda_{i j} u\right\rangle+2 \nabla \varphi_{i j}\left\langle\Delta u, \Lambda_{i j} \nabla u\right\rangle\right) d t d x \\
& =0
\end{aligned}
$$

where two terms vanish because of the skew-symmetry of $\Lambda_{i j}$. For the normal component, we compute

$$
\begin{aligned}
& \int_{\mathbb{R} \times \mathbb{R}^{n}}\left(-\left\langle\partial_{t} u, \partial_{t}(\langle\phi, u\rangle u)\right\rangle+\langle\Delta u, \Delta(\langle\phi, u\rangle u)\rangle\right) d t d x \\
& =\int_{\mathbb{R} \times \mathbb{R}^{n}}\left(-\left|\partial_{t} u\right|^{2}\langle u, \phi\rangle+|\Delta u|^{2}\langle u, \phi\rangle+2\langle\Delta u, \nabla u\rangle \nabla\langle\phi, u\rangle+\langle\Delta u, u\rangle \Delta\langle\phi, u\rangle\right) d t d x \\
& =\int_{\mathbb{R} \times \mathbb{R}^{n}}\left(\left(|\Delta u|^{2}-\left|\partial_{t} u\right|^{2}\right)\langle u, \phi\rangle-|\nabla u|^{2} \Delta\langle u, \phi\rangle+2\langle\Delta u, \nabla u\rangle \nabla\langle u, \phi\rangle\right) d t d x
\end{aligned}
$$

since $\left\langle\partial_{t} u, u\right\rangle=0$ and $\langle\Delta u, u\rangle=-|\nabla u|^{2}$. Summing up, the decomposition (2.2) implies that $u$ solves (1.2) weakly.

(2) $\Rightarrow(1)$. Let $u$ be a weak solution of (1.2). For test functions $v \in W$ taking values in $T_{u(t, x)} S^{l}$ equation (1.4) follows from (1.5) since then $\langle u, v\rangle=0$. 
Note that the conservation law can also be obtained via Noether's theorem. For any map $u$ the action functional $\Phi$ is invariant under rotations $R(\tau) u=\exp (\tau \Lambda) u$. This fact implies

$$
\begin{aligned}
0= & \left.\frac{\mathrm{d}}{\mathrm{d} \tau} \Phi(R(\tau) u, \Omega)\right|_{\tau=0}=\int_{\Omega}\left(\left\langle\partial_{t} u, \partial_{t}(\Lambda u)\right\rangle-\langle\Delta u, \Delta(\Lambda u)\rangle\right) d t d x \\
= & \int_{\Omega}\left(\partial_{t}\left\langle\partial_{t} u, \Lambda u\right\rangle+\Delta\langle\Delta u, \Lambda u\rangle-2 \operatorname{div}\langle\Delta u, \Lambda \nabla u\rangle\right) d t d x \\
& -\int_{\Omega}\left\langle\left(\partial_{t}^{2}+\Delta^{2}\right) u, \Lambda u\right\rangle d t d x .
\end{aligned}
$$

for each subset $\Omega \subset \mathbb{R} \times \mathbb{R}^{n}$. The second integral vanishes since $\left(\partial_{t}^{2}+\Delta^{2}\right) u \perp T_{u} S^{l}$, and hence we have derived again the conservation law (2.1).

Remark 2.2. Similar to Lemma 2.1 one can prove that a (smooth) map $u$ is a solution of (1.6) iff for all $\Lambda \in \mathbb{R}^{(l+1) \times(l+1)}$ with $\Lambda^{T}=-\Lambda$ we have

$$
0=\partial_{t}\left\langle\partial_{t} u, \Lambda u\right\rangle+\Delta\langle\Delta u, \Lambda u\rangle-2 \operatorname{div}\langle\Delta u, \Lambda \nabla u\rangle+2 \operatorname{div}\left\langle|\nabla u|^{2} \nabla u, \Lambda u\right\rangle .
$$

\section{Existence of a global Weak solution}

In this section we construct a global weak solution of (1.2) using a penalization method as in [2]. To this end, we fix an increasing function $\chi \in C^{\infty}([0, \infty))$ with $\chi(s)=1$ for all $s \geq \frac{1}{2}$ and $\chi(s)=s$ for all $s \leq \frac{1}{4}$. We then define the smooth map $F: \mathbb{R}^{l+1} \rightarrow \mathbb{R}$ by

$$
F(x)=\chi \circ\left(|x|^{2}-1\right)^{2} .
$$

Observe that $F$ is bounded, its derivatives are compactly supported, $F^{-1}(0)=S^{l}$, and $\nabla F(x)=0$ if $|x|=1$.

For $\varepsilon>0$ and initial functions $\left(u_{0}, u_{1}\right) \in L^{\infty}\left(\mathbb{R}^{n}, \mathbb{R}^{l+1}\right) \times L^{2}\left(\mathbb{R}^{n}, \mathbb{R}^{l+1}\right)$ with $\Delta u_{0} \in L^{2}\left(\mathbb{R}^{n}, \mathbb{R}^{l+1}\right)$ and $u_{0}(x) \in S^{l}$ for almost every $x \in \mathbb{R}^{n}$, we look at the auxiliary system

$$
\begin{aligned}
& \partial_{t}^{2} u_{\varepsilon}+\Delta^{2} u_{\varepsilon}+\frac{1}{\varepsilon} \nabla F\left(u_{\varepsilon}\right)=0 \\
& u_{\varepsilon}(0, \cdot)=u_{0}, \quad \partial_{t} u_{\varepsilon}(0, \cdot)=u_{1},
\end{aligned}
$$

without requiring that $u(t, x) \in S^{l}$ if $t \neq 0$ a.e.. We point out that the initial value $u_{0}$ here (and below) is not square-integrable, which causes technical difficulties. In contrast to the wave map case in [2], solutions of (3.1) do not possess finite speed of propagation so that standard cut-off arguments cannot be used. Instead we look for (distributional) solutions of the form $u_{\varepsilon}=u_{0}+v_{\varepsilon}$ for a function $v_{\varepsilon}$ solving the shifted system

$$
\begin{aligned}
\partial_{t}^{2} v_{\varepsilon}+\Delta^{2} v_{\varepsilon}+\frac{1}{\varepsilon} \nabla F\left(u_{0}+v_{\varepsilon}\right)+\Delta^{2} u_{0} & =0 \\
v_{\varepsilon}(0, \cdot)=0, \quad \partial_{t} v_{\varepsilon}(0, \cdot) & =u_{1},
\end{aligned}
$$

weakly, with test functions in $W$. For brevity, we sometimes write $H^{k}$ instad of $H^{k}\left(\mathbb{R}^{n}, \mathbb{R}^{l+1}\right)$ for $k \in \mathbb{Z}$, and analogously for other function spaces.

We use the following fact. Let $Z \hookrightarrow Y$ be reflexive Banach spaces, $Z$ be dense in $Y$, and $f: \mathbb{R} \rightarrow Y$ be a weakly continuous function which is essentially bounded with values in $Z$. Then $f$ is bounded and weakly continuous as a map into $Z$. 
Lemma 3.1. Let $\varepsilon>0$ and $\left(u_{0}, u_{1}\right)$ belong to $L^{\infty}\left(\mathbb{R}^{n}, \mathbb{R}^{l+1}\right) \times L^{2}\left(\mathbb{R}^{n}, \mathbb{R}^{l+1}\right)$ with $\Delta u_{0} \in L^{2}\left(\mathbb{R}^{n}, \mathbb{R}^{l+1}\right)$ and $u_{0}(x) \in S^{l}$ for almost every $x \in \mathbb{R}^{n}$. Then there is a distributional solution $u_{\varepsilon}=u_{0}+v_{\varepsilon}$ of $(3.1)$ such that $\Delta v_{\varepsilon}, \partial_{t} v_{\varepsilon} \in L^{\infty}\left(\mathbb{R}, L^{2}\left(\mathbb{R}^{n}, \mathbb{R}^{l+1}\right)\right)$, the functions $v_{\varepsilon}: \mathbb{R} \rightarrow H^{2}\left(\mathbb{R}^{n}, \mathbb{R}^{l+1}\right)$ and $\partial_{t} v_{\varepsilon}: \mathbb{R} \rightarrow L^{2}\left(\mathbb{R}^{n}, \mathbb{R}^{l+1}\right)$ are weakly continuous, and $v_{\varepsilon}$ solves (3.2) weakly with test functions in $W$. For all $t \in \mathbb{R}$, we have the energy inequality

$$
\begin{aligned}
E_{\varepsilon}\left[u_{\varepsilon}\right](t) & :=\int_{\{t\} \times \mathbb{R}^{n}}\left(\frac{1}{2}\left|\partial_{t} u_{\varepsilon}\right|^{2}+\frac{1}{2}\left|\Delta u_{\varepsilon}\right|^{2}+\frac{1}{\varepsilon} F\left(u_{\varepsilon}\right)\right) d x \\
& \leq E_{\varepsilon}\left[u_{\varepsilon}\right](0)=\frac{1}{2} \int_{\mathbb{R}^{n}}\left(\left|u_{1}\right|^{2}+\left|\Delta u_{0}\right|^{2}\right) d x=: E_{0} .
\end{aligned}
$$

Proof. 1) To construct the function $v=u_{\varepsilon}-u_{0}$, we first study a regularized problem (and we drop the subscript $\varepsilon$ ). Let $u_{0}$ and $u_{1}$ be the given data. By means of standard mollifiers, we obtain functions $u_{1, i}$ in $H^{2}$ converging to $u_{1}$ in $L^{2}$ as $i \rightarrow \infty$, as well as $u_{0, j} \in L^{\infty}$ such that $\Delta u_{0, j}$ and $\Delta^{2} u_{0, j}$ belong to $L^{2}$, the maps $u_{0, j}$ tend to $u_{0}$ pointwise a.e. and with a uniform bound, and $\left(\Delta u_{0, j}\right)$ converges to $\Delta u_{0}$ in $L^{2}$ as $j \rightarrow \infty$. Finally, let $\alpha_{k}$ be the characteristic function of the ball $B(0, k)$ in $\mathbb{R}^{n}$. We now introduce the modified equation

$$
\begin{aligned}
\partial_{t}^{2} v+\Delta^{2} v+\frac{1}{\varepsilon} \alpha_{k} \nabla F\left(u_{0, j}+v\right)+\Delta^{2} u_{0, j} & =0 \\
v(0, \cdot)=0, \quad \partial_{t} v(0, \cdot) & =u_{1, i} .
\end{aligned}
$$

Define $X=H^{2}\left(\mathbb{R}^{n}, \mathbb{R}^{l+1}\right) \times L^{2}\left(\mathbb{R}^{n}, \mathbb{R}^{l+1}\right)$. We have $\left(v(t), \partial_{t} v(t)\right) \in X$ and we look at the operator matrix

$$
A=\left(\begin{array}{cc}
0 & -I \\
\Delta^{2} & 0
\end{array}\right), \quad D(A)=H^{4}\left(\mathbb{R}^{n}, \mathbb{R}^{l+1}\right) \times H^{2}\left(\mathbb{R}^{n}, \mathbb{R}^{l+1}\right) \subset X .
$$

Using the group version of the Lumer-Phillips theorem, see Corollary II.3.6 of [1], one checks that $-A$ generates a strongly continuous (unbounded) group. Moreover, the map

$$
G: X \rightarrow X, G(\varphi, \psi)=\left(\begin{array}{c}
0 \\
\frac{1}{\varepsilon} \alpha_{k} \nabla F\left(u_{0, j}+\varphi\right)+\Delta^{2} u_{0, j}
\end{array}\right)
$$

is globally Lipschitz and $C^{1}$. (For the differentiability one can employ the Sobolev embedding $H^{2} \hookrightarrow L^{p}$ for some $p>2$.). Slight variants of Theorems 6.1.2 and 6.1.5 in [5] hence provide a unique global solution $v=v_{i, j, k} \in C\left(\mathbb{R}, H^{4}\right) \cap C^{1}\left(\mathbb{R}, H^{2}\right) \cap$ $C^{2}\left(\mathbb{R}, L^{2}\right)$ of the system (3.4) in this case. We can now differentiate the energy

$$
\tilde{E}_{j, k}[v](t):=\int_{\{t\} \times \mathbb{R}^{n}}\left(\frac{1}{2}\left|\partial_{t} v\right|^{2}+\frac{1}{2}\left|\Delta\left(u_{0, j}+v\right)\right|^{2}+\frac{1}{\varepsilon} \alpha_{k} F\left(u_{0, j}+v\right)\right) d x
$$

with respect to $t \in \mathbb{R}$. Integration by parts yields

$$
\begin{aligned}
\partial_{t} \tilde{E}_{j, k}[v](t) & =\int_{\{t\} \times \mathbb{R}^{n}}\left(\left\langle\partial_{t} v, \partial_{t t} v\right\rangle+\left\langle\Delta^{2}\left(u_{0, j}+v\right), \partial_{t} v\right\rangle+\left\langle\frac{1}{\varepsilon} \alpha_{k} \nabla F\left(u_{0, j}+v\right), \partial_{t} v\right\rangle\right) d x \\
& =0 \\
\tilde{E}_{j, k}[v](t) & =\int_{\mathbb{R}^{n}}\left(\frac{1}{2}\left|u_{1, i}\right|^{2}+\frac{1}{2}\left|\Delta u_{0, j}\right|^{2}+\frac{1}{\varepsilon} \alpha_{k} F\left(u_{0, j}\right)\right) d x
\end{aligned}
$$

for all $t \in \mathbb{R}$. In the next steps, we perform the limits $i, j, k \rightarrow \infty$ one after the other. We will not relabel subsequences. 
2) As in Theorem 6.1.2 of [5], the solution $\left(v, \partial_{t} v\right)$ of (3.4) depends continuously in $X$ on the initial data. The sequence $\left(v_{i, j, k}\right)_{i}$ thus tends in $C\left(\mathbb{R}, H^{2}\right) \cap C^{1}\left(\mathbb{R}, L^{2}\right)$ to a function $v_{j, k}$ for all $j, k \in \mathbb{N}$, and a subsequence also converges pointwise a.e. in $(t, x)$. Note that, here and throughout the paper, the space $C(\mathbb{R}, X)$ (for a normed space $X$ ) consists of all continuous functions $v: \mathbb{R} \rightarrow X$, and convergence therein refers to locally uniform convergence (similarly for $C^{k}(\mathbb{R}, X)$ ). Consequently, the map $v_{j, k}$ satisfies the initial conditions $v_{j, k}(0, \cdot)=0$ and $\partial_{t} v_{j, k}(0, \cdot)=u_{1}$, and it solves the PDE in (3.4) weakly with test functions in $W$.

For a fixed $t \in \mathbb{R}$, a further subsequence $\left(v_{i, j, k}(t, \cdot)\right)_{i}$ tends to $v_{j, k}(t, \cdot)$ a.e.. Hence, the above energy equality leads to the identity

$$
\tilde{E}_{j, k}\left[v_{j, k}\right](t)=\int_{\mathbb{R}^{n}}\left(\frac{1}{2}\left|u_{1}\right|^{2}+\frac{1}{2}\left|\Delta u_{0, j}\right|^{2}+\frac{1}{\varepsilon} \alpha_{k} F\left(u_{0, j}\right)\right) d x=: \tilde{E}_{0}^{j, k}
$$

for all $j, k \in \mathbb{N}$ and $t \in \mathbb{R}$.

3) Now, we pass to the limit $j \rightarrow \infty$ for each fixed $k \in \mathbb{N}$. Because of the cut-off $\alpha_{k}$ and $F\left(u_{0}\right)=0$, the energies $\tilde{E}_{0}^{j, k}$ tend to

$$
E_{0}:=\frac{1}{2} \int_{\mathbb{R}^{n}}\left(\left|u_{1}\right|^{2}+\left|\Delta u_{0}\right|^{2}\right) d x,
$$

so that $\tilde{E}_{j, k}\left[v_{j, k}\right](t)$ is dominated by a number $c_{k}$ for all $j \in \mathbb{N}$ and $t \in \mathbb{R}$. This estimate leads to the convergence

$$
\Delta v_{j, k} \rightarrow \varphi_{k} \quad \text { weak }^{*} \text { in } L^{\infty}\left(\mathbb{R} ; L^{2}\right) \quad \text { and } \quad \partial_{t} v_{j, k} \rightarrow \psi_{k} \quad \text { weak }{ }^{*} \text { in } L^{\infty}\left(\mathbb{R} ; L^{2}\right)
$$

as $j \rightarrow \infty$. The functions $\varphi_{k}$ and $\psi_{k}$ inherit the energy bound by $E_{0}$. We further obtain the estimate

$$
\left\|v_{j, k}(t)\right\|_{L^{2}}=\left\|\int_{0}^{t} \partial_{s} v_{j, k}(s) d s\right\|_{L^{2}} \leq 2 c_{k}^{1 / 2}|t| \leq 2 m c_{k}^{1 / 2}
$$

for all $t \in[-m, m]$ and $j, k \in \mathbb{N}$. The sequence $\left(v_{j, k}\right)_{j}$ is thus bounded in the spaces $L^{\infty}\left(J ; H^{2}\right)$ and $W^{1, \infty}\left(J ; L^{2}\right)$ for each $k \in \mathbb{N}$ and each bounded interval $J \subseteq \mathbb{R}$. Proposition 1.1.4 in [4] implies the interpolative embedding

$$
L^{\infty}\left(J ; H^{2}\right) \cap W^{1, \infty}\left(J ; L^{2}\right) \hookrightarrow C^{1-\beta}\left(J ; H^{2 \beta}\right)
$$

for $\beta \in(0,1)$. So, by the Arzelà-Ascoli theorem, $\left(v_{j, k}\right)_{j}$ tends to a function $v_{k}$ strongly in $C\left(J ; H_{\mathrm{loc}}^{\theta}\right)$ for each $\theta \in[0,2)$ and hence in $C\left(\mathbb{R} ; H_{\mathrm{loc}}^{\theta}\right)$ and pointwise a.e., for a diagonal sequence. A standard test function argument then yields that $\varphi_{k}=\Delta v_{k}$ and $\psi_{k}=\partial_{t} v_{k}$. In particular, $v_{k}$ belongs to $L^{\infty}\left(J ; H^{2}\right) \cap W^{1, \infty}\left(J ; L^{2}\right) \cap$ $C\left(\mathbb{R} ; H^{\theta}\right), v_{k}(0)=0$, and $\Delta v_{k}$ is weakly continuous with values in $L^{2}$. Moreover, it satisfies the energy inequality

$$
\tilde{E}_{k}\left[v_{k}\right](t):=\int_{\{t\} \times \mathbb{R}^{n}}\left(\frac{1}{2}\left|\partial_{t} v_{k}\right|^{2}+\frac{1}{2}\left|\Delta\left(u_{0}+v_{k}\right)\right|^{2}+\frac{1}{\varepsilon} \alpha_{k} F\left(u_{0}+v_{k}\right)\right) d x \leq E_{0}
$$

for all $t \in \mathbb{R}$ and $k \in \mathbb{N}$.

Since the nonlinear term has compact support in space, we next deduce that $v_{k}$ satisfies the PDE in (3.4) for $u_{0}$ instead of $u_{0, j}$ weakly with test functions in $W$. This equation further shows that the weak derivative $\partial_{t}^{2} v_{k}$ actually belongs to $L_{\text {loc }}^{\infty}\left(\mathbb{R} ; H^{-2}\right)$ so that $\partial_{t} v_{k}$ is continuous from $\mathbb{R}$ to $H^{-2}$ and, as seen above, essentially bounded in $L^{2}$. As a result, the map $t \mapsto \partial_{t} v_{k}(t)$ is bounded and weakly continuous in $L^{2}$. Since $\partial_{t} v_{j, k}$ converges weak ${ }^{*}$ in $L^{\infty}\left(\mathbb{R} ; L^{2}\right)$ and vanishes at $t=0$, we conclude that $\partial_{t} v_{k}(0)=0$. 
4) In a final step, we let $k \rightarrow \infty$. We can proceed as in Step 3) to construct a limit function $v$ with the desired properties. There is only one difference in the derivation of the PDE for $v$. To apply the dominated convergence theorem, observe that $\left|\nabla F\left(u_{0}+v_{k}\right)\right|$ is bounded by $c\left|v_{k}\right|$ and that a converging sequence in $L^{2}$ has a subsequence with a majorant in $L^{2}$. Finally, the function $u_{\varepsilon}=u_{0}+v$ satisfies the assertions.

Based on the energy estimate (3.3), we can now pass to the limit $\varepsilon \rightarrow 0$ in (3.1). The special form of the penalization term implies that the resulting weak limit $u$ takes values in $S^{l}$. As in [2], we employ the equation (2.1) in divergence form to show that $u$ indeed solves of (1.2) weakly. To identify its initial values, we have to assume that $u_{1}$ maps into the tangent space of $S^{l}$.

Proof of Theorem 1.1. 1) We use the functions $u_{j}=u_{0}+v_{j}$ from Lemma 3.1, where $v_{j}=v_{\varepsilon_{j}}$ for some $\varepsilon_{j} \rightarrow 0^{+}$. Let $\Lambda \in \mathbb{R}^{(l+1) \times(l+1)}$ be skew-symmetric and $\varphi \in C_{c}^{\infty}\left(\mathbb{R} \times \mathbb{R}^{n}\right)$. We take $\varphi \Lambda u_{j}$ as a test function for (3.1). (It does not belong to $V$, in general, but the regularity provided by Lemma 3.1 suffices here.) Since $\nabla F\left(u_{j}\right)$ is a scalar multiple of $u_{j}$, we can argue as in the first part of the proof of Lemma 2.1 and conclude that $u_{j}$ fulfills the equation

$$
0=\partial_{t}\left\langle\partial_{t} u_{j}, \Lambda u_{j}\right\rangle+\Delta\left\langle\Delta u_{j}, \Lambda u_{j}\right\rangle-2 \operatorname{div}\left\langle\Delta u_{j}, \Lambda \nabla u_{j}\right\rangle .
$$

in the distributional sense.

2) Starting from the energy estimate (3.3), we can next pass to the limit $\varepsilon_{j} \rightarrow 0$ as in Step 3) of the proof of Lemma 3.1 (again without relabelling subsequences). The functions $v_{j}$ then converge strongly in $C\left(\mathbb{R} ; H_{\text {loc }}^{\theta}\right)$ for $\theta<2$ and pointwise a.e. to a map $v \in C\left(\mathbb{R} ; H^{\theta}\right)$. Moreover, $\partial_{t} v_{j}$ and $\Delta v_{j}$ tend to $\partial_{t} v$ and $\Delta v$ weak* in $L^{\infty}\left(\mathbb{R}, L^{2}\right)$. Combining these facts, we infer that $\Delta v: \mathbb{R} \rightarrow L^{2}$ is bounded and weakly continuous. The limit $u:=u_{0}+v$ thus satisfies $u(0, \cdot)=u_{0}$ and $E[u](t) \leq E_{0}$ for all $t \in \mathbb{R}$. Thanks to (3.6) and the convergence of $v_{j}$, the function $u$ solves (2.1) distributionally.

The energy bound (3.3) further says that $\left\|F\left(u_{j}(t, \cdot)\right)\right\|_{1} \leq \varepsilon_{j} E_{0}$ for all $j \in \mathbb{N}$ and $t \in \mathbb{R}$. For each bounded interval $J \subseteq \mathbb{R}$, Fatou's Lemma now implies that

$$
\int_{J \times \mathbb{R}^{n}} F(u) d x d t \leq \liminf _{j \rightarrow \infty} \int_{J \times \mathbb{R}^{n}} F\left(u_{j}\right) d x d t=0 .
$$

Hence, $F(u)=0$ and therefore $u(t, x) \in S^{l}$ for a.e. $(t, x) \in \mathbb{R} \times \mathbb{R}^{n}$. The continuity of $u-u_{0}: \mathbb{R} \rightarrow L^{2}$ then implies that $u(t, x)$ belongs $S^{l}$ for each $t \in \mathbb{R}$ and a.e. $x \in \mathbb{R}^{n}$. Since $u \in Z$, the map $\nabla u$ is contained $L^{\infty}\left(\mathbb{R}, L^{4}\right)$ by (1.3). We can now deduce that $u$ weakly solves (2.1) with test functions in $W_{s}$, and so $u$ is a weak solution of (1.2) by Lemma 3.1. Moreover, the equation $|u|^{2}=1$ yields $\left\langle\partial_{t} u, u\right\rangle=0$ so that $\partial_{t} u(t, x)$ is contained in the tangent space $T_{u(t, x)} S^{l}$ for a.e. $(t, x)$.

3) We still have to show the continuity of $\partial_{t} u, \Delta u: \mathbb{R} \rightarrow L^{2}$ and that $\partial_{t} u(0, \cdot)=u_{1}$. So far we know that the first map is essentially bounded. Let $\Lambda \in \mathbb{R}^{(l+1) \times(l+1)}$ again be skew-symmetric. The equation (2.1) and the above stated regularity properties of $u$ imply that $\partial_{t}\left\langle\partial_{t} u, \Lambda u\right\rangle$ is bounded in $H^{-2}+W^{-1,4 / 3}$. Hence, the function $\psi: t \mapsto\left\langle\partial_{t} u, \Lambda u\right\rangle$ is continuous in this space. Consequently, $\psi$ is bounded and weakly continuous in $L^{2}$. Step 2) implies that for a.e. $t \in \mathbb{R}$ the vector $\partial_{t} u(t, x)$ belongs to $T_{u(t, x)} S^{l}$ for a.e. $x \in \mathbb{R}^{n}$. In view of $(2.2)$, by modifying $\partial_{t} u(t, \cdot)$ for $t$ in set of measure 0 we obtain a representative $\partial_{t} u$ which is bounded and weakly continuous as a map from $\mathbb{R}$ to $L^{2}$. 
Next, we multiply the equations (3.6) for $u_{j}$ and (2.1) for $u$ by a function $\varphi \in$ $C_{c}^{\infty}\left(\mathbb{R} \times \mathbb{R}^{n}\right)$. We integrate by parts in $t \in[0, \infty)$ with values in $H^{-2}+W^{-1,4 / 3}$ and subtract the two resulting equations, which yields

$$
\begin{aligned}
\int_{\{0\} \times \mathbb{R}^{n}}\left\langle u_{1}-e \partial_{t} u, \Lambda u_{0}\right\rangle \varphi d x= & \int_{0}^{\infty} \int_{\mathbb{R}^{n}}\left(-\left\langle\partial_{t} u_{j}, \Lambda u_{j}\right\rangle+\left\langle\partial_{t} u, \Lambda u\right\rangle\right) \partial_{t} \varphi d x d t \\
& +\int_{0}^{\infty} \int_{\mathbb{R}^{n}}\left(\left\langle\Delta u_{j}, \Lambda u_{j}\right\rangle-\langle\Delta u, \Lambda u\rangle\right) \Delta \varphi d x d t \\
& \left.+2 \int_{0}^{\infty} \int_{\mathbb{R}^{n}}\left(\left\langle\Delta u_{j}, \Lambda \nabla u_{j}\right\rangle-\langle\Delta u, \Lambda \nabla u\rangle\right\rangle\right) \nabla \varphi d x d t .
\end{aligned}
$$

By Step 2), the right hand side converges to zero as $j \rightarrow \infty$ so that

$$
\left\langle\partial_{t} u(0)-u_{1}, \Lambda u_{0}\right\rangle=0 .
$$

As both $u_{1}$ and $\partial_{t} u(0)$ belong to $T_{u_{0}} S^{l}$ a.e., we conclude that $\partial_{t} u(0)=u_{1}$.

Finally, in the energy bound obtained in Step 2) we can replace the initial time 0 by any $t \in \mathbb{R}$ since $u(t, \cdot)$ and $\partial_{t} u(t, \cdot)$ fulfill the conditions for the initial data and hence

$$
E[u](0) \leq E[u](t) \leq E[u](0)
$$

which implies the energy identity. Further, in conjunction with the weak continuity, this identity implies the strong continuity of $\partial_{t} u, \Delta u: \mathbb{R} \rightarrow L^{2}$.

\section{REFERENCES}

1. Klaus-Jochen Engel and Rainer Nagel, One-parameter semigroups for linear evolution equations, Graduate Texts in Mathematics, vol. 194, Springer-Verlag, New York, 2000. MR 1721989

2. A. Freire, Global weak solutions of the wave map system to compact homogeneous spaces, Manuscripta Math. 91 (1996), no. 4, 525-533. MR 1421290

3. E. Hebey and B. Pausader, An introduction to fourth order nonlinear wave equations, http://www.math.univ-paris13.fr/ pausader/HebPausSurvey.pdf.

4. Alessandra Lunardi, Analytic semigroups and optimal regularity in parabolic problems, Progress in Nonlinear Differential Equations and their Applications, vol. 16, Birkhäuser Verlag, Basel, 1995. MR 1329547

5. A. Pazy, Semigroups of linear operators and applications to partial differential equations, Applied Mathematical Sciences, vol. 44, Springer-Verlag, New York, 1983. MR 710486

6. Jalal Shatah, Weak solutions and development of singularities of the $\mathrm{SU}(2) \sigma$-model, Comm. Pure Appl. Math. 41 (1988), no. 4, 459-469. MR 933231

7. Jalal Shatah and Michael Struwe, Geometric wave equations, Courant Lecture Notes in Mathematics, vol. 2, New York University Courant Institute of Mathematical Sciences, New York, 1998. MR 1674843 (2000i:35135)

(S. Herr) Fakultät für Mathematik, Universität Bielefeld, Postfach 1001 31, 33501 Bielefeld, Germany

E-mail address: herr@math.uni-bielefeld.de

(T. Lamm) Department of Mathematics, Karlsruhe Institute of Technology, 76128 Karlsruhe, Germany

E-mail address: tobias.lamm@kit.edu

(R. Schnaubelt) Department of Mathematics, Karlsruhe Institute of Technology, 76128 Karlsruhe, Germany

E-mail address: schnaubelt@kit.edu 